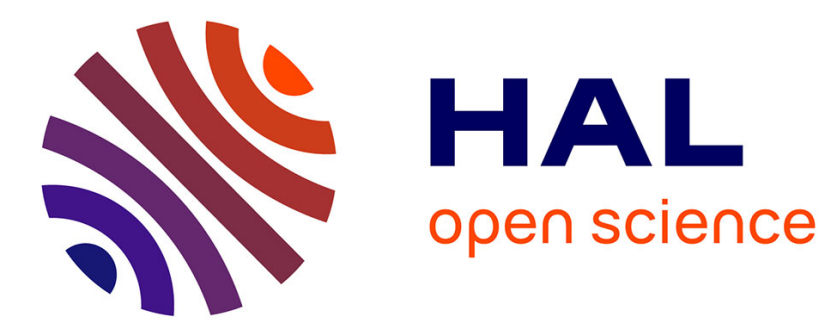

\title{
Les actes de parole de l'enseignant: déterminants et révélateurs de sa pratique
}

\author{
Eric Roditi
}

\section{To cite this version:}

Eric Roditi. Les actes de parole de l'enseignant: déterminants et révélateurs de sa pratique. Spirale - Revue de Recherches en Éducation , 2014, 54, pp.85-101. halshs-01059376

\section{HAL Id: halshs-01059376 \\ https://shs.hal.science/halshs-01059376}

Submitted on 17 Dec 2021

HAL is a multi-disciplinary open access archive for the deposit and dissemination of scientific research documents, whether they are published or not. The documents may come from teaching and research institutions in France or abroad, or from public or private research centers.
L'archive ouverte pluridisciplinaire HAL, est destinée au dépôt et à la diffusion de documents scientifiques de niveau recherche, publiés ou non, émanant des établissements d'enseignement et de recherche français ou étrangers, des laboratoires publics ou privés. 


\section{Les actes de parole de l'enseignant : déterminants et révélateurs}

\section{de sa pratique}

\section{Éric Roditi}

\section{Abstract}

This article uses three pieces of research in the field of mathematics education in order to offer several ways to analyse teachers'practices by analysing teachers' discourses. Its theoretical framework leads to consider teachers'discourses as an ingredient rather than a product of teaching practices. Although linguistic concepts and tools can help to offer a certain perspective, the central issue is to understand how teaching practices contribute to pupils' learning.

\section{Résumé}

À travers trois exemples de recherche en didactique des mathématiques sur les pratiques d'enseignants, cet article propose différentes analyses de leurs discours tenus en classe. L'option théorique sous-jacente conduit à considérer que ces discours traversent leurs pratiques et non qu'elles en seraient le produit. L'analyse des actes de paroles des enseignants contribuent alors à celle de leur pratique. Elle convoque, plus ou moins directement, des outils construits en linguistique, même si la perspective didactique mène à considérer d'abord les pratiques pour les apprentissages mathématiques auxquelles elles conduisent.

\section{Citer ce document / Cite this document :}

Roditi Éric. Les actes de parole de l'enseignant : déterminants et révélateurs de sa pratique. In: Spirale. Revue de recherches en éducation, $n^{\circ} 54,2014$. Langage, apprentissage et enseignement des mathématiques. pp. 85-101;

http://www.persee.fr/doc/spira_0994-3722_2014_num_54_1_1038

Document généré le 20/10/2017 


\title{
LES ACTES DE PAROLE \\ DE L'ENSEIGNANT : \\ DÉTERMINANTS ET RÉVÉLATEURS \\ DE SA PRATIQUE
}

\begin{abstract}
Résumé : À travers trois exemples de recherche en didactique des mathématiques sur les pratiques d'enseignants, cet article propose différentes analyses de leurs discours tenus en classe. L'option théorique sous-jacente conduit à considérer que ces discours traversent leurs pratiques et non qu'elles en seraient le produit. L'analyse des actes de paroles des enseignants contribuent alors à celle de leur pratique. Elle convoque, plus ou moins directement, des outils construits en linguistique, même si la perspective didactique mène à considérer d'abord les pratiques pour les apprentissages mathématiques auxquelles elles conduisent.
\end{abstract}

Mots-clés : pratiques enseignantes, nombres décimaux, géométrie, acte de parole, double approche didactique et ergonomique.

L'analyse du langage de l'enseignant pour l'étude de sa pratique, tel est l'objet développé dans cet article à travers trois recherches en didactique des mathématiques. En première approche, nous pourrions nous reconnaître aisément parmi les didacticiens pour lesquels l'analyse des pratiques langagières constitue un outil pour interroger d'autres phénomènes, notamment la contribution de l'enseignant à la construction de connaissances mathématiques par les élèves. Pourtant, nos orientations théoriques, globalement inspirées de la théorie de l'activité, nous amènent à concevoir les activités des enseignants comme étant constitutives de leur pratique. Nous dirons donc plutôt que nous étudions les pratiques langagières des enseignants en tant qu'elles sont à la fois déterminantes et révélatrices de leur pratique. C'est ce que nous nous proposons de montrer dans ce texte où nous présentons d'abord nos choix théoriques et nos outils méthodologiques généraux relatifs à l'analyse des discours des enseignants, et où nous développons ensuite trois exemples dans lesquels les analyses révèlent comment les enseignants agissent, par le langage, tant sur l'enseignement qu'ils dispensent que sur l'apprentissage de leurs élèves.

\section{ACTES DE PAROLE ET ACTIVITÉS DE L'ENSEIGNANT, PRATIQUES ENSEIGNANTES}

En présentant le cadre théorique sous-jacent à nos recherches, nous précisons comment l'expression «pratiques enseignantes », très largement utilisée en éducation, doit être comprise dans ce texte, puis nous indiquons comment les discours des enseignants sont appréhendés.

Différentes activités interdépendantes, une pratique cohérente

Avec une double approche didactique et ergonomique (Robert \& Rogalski 2008 ; Roditi 2013), nous nous référons théoriquement à la didactique des ma-

Spirale - Revue de Recherches en Éducation - $2014 N^{\circ} 54$ (85-101) 
thématiques et à la théorie de l'activité (Leontiev 1984) pour étudier les pratiques enseignantes dans le but de les décrire et de les comprendre. Nous appliquons ainsi, à l'enseignant au travail, la modélisation de l'activité co-déterminée par le sujet et la situation. Concernant le sujet enseignant, nous sommes particulièrement attentifs à ses conceptions de l'enseignement et de l'apprentissage, ainsi qu'à son expérience professionnelle, y compris sa formation. Quant aux situations dans lesquelles il est engagé, nous retenons principalement les tâches qui lui sont attribuées, notamment par les instructions officielles et les programmes scolaires, et le contexte socio-institutionnel dans lequel il doit les accomplir. Conformément aux fonctions assignées au travail des enseignants, nous analysons leurs activités en considérant leur finalité d'instruction, c'est-à-dire de construction de connaissances par les élèves, et d'éducation, c'est-à-dire de transmission de valeurs et de règles de vie sociale.

Cet intérêt pour les activités correspond à notre volonté d'appréhender le travail enseignant d'une manière assez globale, en tenant compte de sa complexité, de la multiplicité de ses fonctions, en ne considérant pas seulement le travail effectué en classe à l'aune des apprentissages visés pour les élèves. Prendre en compte les prescriptions qui sont adressées aux enseignants pour analyser leur activité ne signifie pas supposer qu'ils ne font que chercher à les satisfaire : ils s'en affranchissent parfois, ils agissent aussi indépendamment d'elles, pour faire comme ils pensent devoir faire, ou pour faire comme d'autres font. Enfin, si l'activité de l'enseignant correspond à la fois à ce qu'il fait, ce qu'il pense et ce qu'il ressent, elle recouvre aussi ce qu'il a voulu faire et qu'il a cru faire, ce qu'il aurait pu faire, ce qu'il a délibérément choisi de ne pas faire, ce qu'il aurait voulu faire et qu'il n'a pas pu faire, ce qu'il voulait éviter de faire et qu'il a été obligé de faire, etc.

Bien que les résultats des recherches mentionnés ici ne portent que sur l'enseignement en classe, nous considérons d'autres activités de l'enseignant, principalement la préparation des cours et l'évaluation des apprentissages, mais aussi parfois la coopération avec les autres acteurs du système éducatif. C'est finalement à l'ensemble de ce système d'activités que nous nous référons lorsque nous employons le terme de pratique enseignante. Les recherches sur les pratiques professionnelles invitent à supposer qu'elles sont cohérentes, et cela malgré toutes les adaptations nécessaires aux contextes qui engendrent une variabilité des activités. En ce qui concerne les enseignants, on peut notamment penser aux travaux menés autour de Robert: Peltier-Barbier (2004), Roditi (2005) et Vandebrouck (2008). Nos travaux visent à étayer cette hypothèse de cohérence, notamment par la mise au jour de logiques personnelles et professionnelles qui sous-tendent les activités. Aussi préférons-nous utiliser le terme «pratique » au singulier lorsqu'il s'agit de celle d'un même enseignant.

Les actes de parole et leur analyse

Les analyses des discours tenus en classe par l'enseignant contribuent à l'étude de ce qu'il met en œuvre, notamment par le langage, pour réaliser son projet d'enseignement et l'adapter en fonction des élèves ou d'impératifs, par exemple temporels, auxquels il doit répondre. Elles contribuent également, par triangulation, à valider des hypothèses émises sur sa pratique à partir d'autres analyses portant sur les contenus enseignés, sur les tâches proposées et les modifications qui y sont apportées en classe, ainsi que sur les entretiens conduits avant et après les séances observées. 
D'un point de vue méthodologique, les enseignements observés sont enregistrés et transcrits. Ils sont d'abord décomposés en séances, c'est-à-dire en unités thématiques relativement à un contenu, puis en épisodes correspondant à chacune des tâches mathématiques prescrites aux élèves. Précisons que dans ce texte, le terme de tâche doit être entendu au sens qui lui est accordé en psychologie ergonomique (Leplat 1997) ; pour les élèves, nous l'utiliserons généralement sans préciser qu'il s'agit d'une tâche mathématique prescrite par l'enseignant, sans préciser non plus comment cette tâche est redéfinie puisque c'est plutôt sur l'enseignant et sa pratique que portent nos analyses. Le découpage de l'enseignement en séances et en épisodes permet d'analyser les activités de l'enseignant et des élèves en référence à de nombreux outils produits en didactique des mathématiques.

Les transcriptions sont enfin décomposées en portions délimitées par les changements de locuteurs, les moments de silence ou les pauses du discours. Ces portions possèdent une unité de signification, elles sont considérées comme des actes de parole. L'expression « acte de langage » se réfère à la théorie du philosophe Austin (1970), reprise par Searle (1972), qui insiste sur le fait que la langue ne permet pas seulement de décrire le monde, mais aussi d'agir sur lui. Ainsi, dans le contexte de l'enseignement des mathématiques, cette inspiration correspond au fait que, dans nos analyses, les discours des enseignants ne sont pas seulement considérés pour les savoirs exprimées dans le but de les transmettre, mais aussi pour toutes les interventions dont le but est d'agir sur leur classe ou sur leurs élèves, et même de les faire (ré-)agir pour qu'ils construisent les connaissances visées. Ce dernier aspect est caractéristique de l'approche interactionniste à laquelle Kerbrat-Orecchioni (1990) a largement contribué en développant de nombreux outils pour l'analyse des interactions langagières. Comme indiqué dans l'introduction, le langage verbal oral seulement est analysé dans les recherches présentées dans cet article, même s'il arrive parfois que les actes de langage considérés soient écrits, au tableau par exemple. Les portions de discours évoquées précédemment sont donc, comme y invite la sémiologue Nuchèze (2001), appelés « actes de parole ».

La recherche en linguistique sur les actes de langage a conduit à distinguer différentes catégories d'actes qui, d'une part, portent les choix théoriques des auteurs concernant le discours ou plus largement le langage, et qui, d'autre part, constituent des outils pour analyser ces discours. Pour une première approche de ces questions, des éléments essentiels sont clairement développés dans le Dictionnaire d'analyse du discours (Chareaudeau \& Maingueneau 2002) à l'article « Acte de langage » et à ceux qui y sont liés. En ce qui nous concerne, les analyses que nous avons cherché à développer des discours de l'enseignant en nous attachant aux actes de paroles ne relèvent ni de la syntaxe - étude interne à la langue portant sur les relations des signes aux autres signes - ni de la sémantique - étude des relations des signes de la langue avec la réalité - mais plutôt de la pragmatique qui s'intéresse aux manières d'utiliser les signes de la langue, en lien avec les utilisateurs et les effets qu'ils peuvent produire. Mais la visée didactique qui est la nôtre nous éloigne sensiblement de l'analyse linguistique dans le sens où nous ne cherchons pas à mettre en lumière comment l'enseignant utilise le discours pour agir, mais seulement ce qu'il cherche à obtenir par le discours. Ainsi, par exemple, une analyse linguistique pourrait conduire à distinguer les actes de parole «Taisez-vous !», « Merci de rester silencieux. » et «Vous ai-je autorisés à parler? ». Ils correspondent en effet à trois signifiants différents, du fait de la forme de l'énoncé qui est respectivement impérative, affirmative et interrogative. Nos analyses étant didactiques, nous choisirions plutôt d'envisager ces trois actes de parole comme étant équivalents, parce que le signifié global est le même, et nous les 
considérerions alors comme trois rappels à l'ordre scolaire. Si notre option théorique sur le discours se réfère explicitement aux sciences du langage, la catégorisation que nous adoptons des actes de parole n'est pas empruntée à la linguistique. Elle découle d'hypothèses et de savoirs propres à la recherche en éducation et plus particulièrement à la didactique des mathématiques. Cela nous permet de rendre compte de l'action du professeur sur le déroulement de l'enseignement et sur les apprentissages de ses élèves. Les choix de catégorisation qui déterminent les analyses dépendent des objectifs de l'étude et donc de la problématique précise de la recherche. Des compléments méthodologiques seront par conséquent apportés pour chacune des trois recherches présentées dans ce texte.

Il faut enfin ajouter que, dans nos recherches, cette conception du langage comme action sur le monde est seulement mis à l'œuvre pour analyser des discours tenus en classe, même si nos travaux reposent à la fois sur des observations de séquences d'enseignement et sur des entretiens avec les enseignants. Ainsi, le corpus discursif étudié et les méthodes convoquées ne visent pas un recouvrement des analyses des pratiques discursives et des analyses des pratiques d'enseignement. Les premières en effet, contrairement aux secondes, ne s'attachent véritablement ni aux contraintes contextuelles et à leur objectivation, ni à l'inscription du sujet enseignant dans sa pratique.

Finalement, notre démarche d'ensemble consiste bien à concevoir la pratique d'un enseignant comme une entité cohérente comprenant des activités de différents types (préparation des cours, enseignement, évaluation, travail collectif, etc.), chacune de ces activités étant co-déterminée par l'enseignant lui-même et par la situation à laquelle il est confronté, chacune de ces activités ayant en retour une influence sur l'enseignant lui-même et sur la situation. Nous pensons de même pour les pratiques discursives : les actes de paroles de l'enseignant en classe traversent ses activités d'enseignement et sont par conséquent appréhendés comme des déterminants de sa pratique, même si, bien sûr, les contextes influencent les activités et donc les discours tenus durant ces activités. Enfin, parce que leurs actes de paroles sont analysés au même titre que d'autres activités, ils constituent des révélateurs de la pratique, comme ces autres activités.

Trois recherches sont développées qui illustrent cette démarche. La première analyse la trajectoire d'un enseignant et son positionnement professionnel entre les missions d'éducation et de transmission des savoirs mathématiques. La deuxième compare les pratiques de quatre enseignants et met en lumière leur manière différente de réguler le déroulement des cours, par le langage, en agissant sur la nature de l'activité mathématique des élèves. La troisième recherche porte sur un dispositif d'aide à la comparaison des nombres décimaux où les élèves sont amenés à travailler sur l'expression écrite et orale de ces nombres, sur leurs représentations concrètes ainsi que sur les règles qu'ils utilisent et leur justification.

\section{LES DISCOURS ET LA PRATIQUE D'UN ENSEIGNANT :} ANALYSE DES DIX PREMIÈRES ANNÉES D'EXERCICE

La première recherche est codisciplinaire, elle rassemble des chercheurs en éducation convoquant chacun une approche particulière : didactique, sociologique ou clinique d'orientation psychanalytique (Chaussecourte 2014). Elle porte sur les évolutions de la pratique d'un enseignant que nous appelons Benoît, et qui a été observé pendant dix années depuis la période où il était stagiaire en formation initiale jusqu'à celle où il est devenu un professeur expérimenté, directeur d'école et futur formateur. Cette recherche a nécessité de nombreux entretiens ain- 
si qu'une dizaine de films de séquences d'enseignement. En ce qui concerne les mathématiques, l'analyse repose sur un ensemble homogène quant aux contenus : ce sont les cinq séances de géométrie filmées durant cette période qui ont été analysées.

L'évolution de la pratique enseignante de Benoît en géométrie

L'analyse didactique qui a été effectuée ne peut être présentée ici, nous indiquons néanmoins les résultats principaux qu'elle a permis d'obtenir afin de montrer comment l'analyse des actes de paroles de Benoît est croisée avec une étude plus globale de ses activités et de sa pratique. Précisons que, dans cette recherche, nous avons tenu compte des travaux très instructifs de Butlen, PeltierBarbier et Pézard (2002) qui pointent, dans les pratiques des débutants, des contradictions auxquelles ils doivent faire face et qui agissent comme des contraintes sur leur pratique. Nous avons par conséquent porté une attention particulière à la tension entre les finalités d'instruction et d'éducation, ainsi qu'aux tâches proposées aux élèves comme à la conduite de leur réalisation en classe par les élèves.

Les objectifs majeurs d'une leçon sont généralement bien compris par l'enseignant, en référence aux prescriptions institutionnelles. Néanmoins, des savoirs précis sur l'apprentissage des contenus en jeu lui manquent souvent, même lors de la dernière séance où il vient d'obtenir le certificat qui lui permettrait de se voir confier des fonctions de formateur. Cela le conduit, de manière assez stable dans le temps, à une programmation peu précise de son enseignement où les difficultés des élèves ne sont guère anticipées. Il manque alors de ressources, en classe, pour les aider à les surmonter et faire qu'ils construisent les connaissances visées.

Une évolution est toutefois perceptible quant à ses objectifs. À ses débuts, il maintient fermement ses visées relatives à la transmission des savoirs, et cela malgré les difficultés qu'il rencontre et qui conduisent à une certaine agitation de la classe. Il met rapidement les élèves au travail, sur des tâches plutôt simples, et l'enseignement se poursuit sous forme de cours dialogué où il évalue les réponses des élèves aux questions posées. Ce schéma se transforme avec l'expérience : Benoît laisse alors plus d'autonomie à ses élèves et il est plus attentif aux difficultés qu'ils expriment, mais cela le conduit à renoncer plus facilement aux finalités d'apprentissage dès que les réactions menacent de devenir trop délicates à gérer. Dans les entretiens, il indique clairement sa volonté de laisser progresser les élèves selon leur rythme et d'obtenir un climat de classe serein.

La finalité éducative de la pratique de Benoît n'a été étudiée qu'à travers ses séances d'enseignement de la géométrie, pourtant, l'analyse a révélé une nette évolution. À ses débuts, le face-à-face caractérise sa relation avec ses élèves qui oscille entre rappels à l'ordre et récompenses. Après quelques années d'exercice, Benoît n'est plus face à ses élèves ; au contraire, il leur propose des tâches à réaliser en équipes, il est à leurs côtés et les accompagne dans leurs activités. Les problèmes didactiques n'ont pas disparu pour autant, néanmoins, comme les savoirs définis par les programmes scolaires sont traités, les critères institutionnels lui permettent d'assumer pleinement sa posture professionnelle et de stabiliser sa pratique : il arrive effectivement à gérer sa classe et à traiter les contenus prescrits.

Les actes de parole de l'enseignant : un élément de sa pratique

Afin de percevoir l'évolution de la pratique de Benoît et la cohérence de cette évolution à travers ses différentes activités, les actes de parole de l'enseignant ont été analysés en tant qu'éléments de cette pratique, les résultats obtenus ont été croisés avec les conclusions des analyses précédentes. 
Pour l'analyse des discours de Benoît, nous avons élaboré des catégories permettant de rendre compte de sa manière de se confronter aux contradictions mises au jour par Butlen, Peltier-Barbier et Pézard et donc de mettre au jour l'évolution éventuelle des moyens mis en œuvre dans sa pratique pour y répondre. La première d'entre elles est une contradiction entre logique de socialisation des élèves et logique des apprentissages; elle explique que, pour chaque séance, les actes de parole de Benoît ont d'abord été classés en deux groupes suivant leur fonction globale : la transmission des savoirs mathématiques ou la conduite de la classe. Les auteurs relevaient également des contradictions entre logique de la réussite immédiate et logique de l'apprentissage, ainsi qu'entre individuel, public et collectif. Ces contradictions conduisent de nombreux enseignants novices à écourter le temps de l'appropriation de la tâche par les élèves au profit d'une succession d'évaluation de leur production et d'aide à la réussite, avec une concentration des interventions individuelles ou publiques et un délaissement quasi systématique des phases collectives de synthèse et d'institutionnalisation. Ces considérations inspirent largement le second niveau de la classification que nous avons élaborée pour l'analyse des actes de paroles. Ceux qui concernent directement la construction des connaissances mathématiques ont été distingués selon quatre fonctions didactiques : $1^{\circ}$ ) les indications et les explications des tâches ; $2^{\circ}$ ) les aides à leur réalisation ; $3^{\circ}$ ) les évaluations des productions des élèves ; $4^{\circ}$ ) les synthèses des connaissances à retenir. Cette catégorisation ne découle pas d'une référence directe à la théorie des situations didactiques, pourtant, ces quatre types d'actes de paroles pourraient correspondre aux contributions de l'enseignant au déroulement des situations de dévolution, d'action et de formulation, de validation et d'institutionnalisation (Brousseau 1998). Aussi proposons-nous, bien que l'acception des termes doive en être élargie par rapport à leur sens initial, de désigner ces quatre types d'actes de paroles comme des actes de dévolution, d'action-formulation, de validation et d'institutionnalisation. Les autres actes de paroles, qui ont pour fonction de garantir le déroulement de la séance, sans lien direct avec la construction des connaissances mathématiques, se répartissent entre des rappels à l'ordre qui portent sur la conduite des élèves, et des interventions qui soutiennent l'organisation du travail en classe.

Comme indiqué dans le dictionnaire d'analyse de discours déjà cité, la notion d'acte de langage laisse encore des problèmes théoriques non résolus, notamment concernant leur délimitation, à cause du continuum qui apparaît d'un acte à l'autre. Dans la transcription des séances, nous avons utilisé des barres obliques pour indiquer les pauses des locuteurs. Pour l'analyse, nous avons utilisé ces barres obliques afin de délimiter les actes de parole. Nous nous sommes en effet rendu compte que ces segments de discours constituaient chacun un signifié bien identifiable, même si deux actes successifs pouvaient viser le même objectif et auraient pu, avec un autre choix méthodologique, être rassemblés en un seul acte au nom du continuum évoqué précédemment.

Sans prétendre épuiser la question méthodologique délicate du codage d'un verbatim, voici, à titre d'exemple du travail effectué pour les cinq séances analysées, un très court extrait où un élève conteste qu'une figure soit un carré, pensant qu'il s'agit d'un rectangle. Benoît l'interrompt et produit quatre actes de parole.

$E d d y$ : non rectangle/parce que là c'est plus grand que là/

Benồt : chut/il dit rectangle parce que/là c'est plus grand que là/qui est-ce qui avait mis carré là/ 
Du point de vue linguistique, le premier acte de parole est une interjection qui a valeur d'ordre, mais ce n'est pas une analyse linguistique que nous menons : la classification présentée précédemment conduit à considérer cet acte de parole comme un rappel à l'ordre scolaire produit par l'enseignant en réaction à l'agitation de la classe. Les deux suivants apparaissent comme des reformulations - pratiquement à l'identique - à l'intention de la classe qui est trop agitée pour mettre au travail les deux affirmations de l'élève Eddy. Malgré le continuum qui peut être constaté entre ces deux actes de paroles et celui qui les précède, nous considérons qu'il y a une forme de rupture dans le discours de Benoît par le fait qu'il porte à présent sur des mathématiques, avec une incitation des élèves à prendre la responsabilité du travail du savoir. Ils ont en ce sens une «valence dévolutive » qui a conduit à les coder comme des actes de dévolution. Le dernier acte de parole est une question adressée à la classe. Sans chercher ici à rendre compte de la stratégie discursive du professeur, comme le ferait sans doute un analyste de discours de l'école française, nous remarquons que Benoît ne soutient plus le travail mathématique des élèves par cette demande concernant les résultats de leur activité passée : la question ne porte pas sur le savoir, mais sur les élèves euxmêmes. Il ne profitera pas du repérage des élèves pour s'engager dans telle ou telle manière de traiter le savoir, il s'agit donc ici, pour l'enseignant, de soutenir le déroulement du cours, ce qui explique le codage de cet acte de parole comme aide au déroulement.

Le verbatim de chaque séance a été ainsi codé. Deux époques ont été distinguées pour mettre en lumière une évolution éventuelle des actes de parole. Les trois premières séances correspondent aux débuts de carrière de l'enseignant, et les deux dernières à sa maturité professionnelle. L'étude porte sur 4986 actes de parole, les informations recueillies figurent dans le tableau $\mathrm{n}^{\circ} 1$. On constate d'emblée la stabilité de la répartition de la parole entre l'enseignant et les élèves : Benoît produit $71 \%$ puis $73 \%$ des actes de parole.

\begin{tabular}{lcc}
\hline & Débuts & Maturité \\
\hline Actes de parole de l'enseignant & $71 \%$ & $73 \%$ \\
\hline Transmission des savoirs (instruction) & $56 \%$ & $61 \%$ \\
Dévolution & $11 \%$ & $20 \%$ \\
Action-Formulation & $29 \%$ & $25 \%$ \\
Validation & $14 \%$ & $10 \%$ \\
Institutionnalisation & $2 \%$ & $6 \%$ \\
\hline Conduite de la classe (éducation) & $44 \%$ & $39 \%$ \\
Rappels à l'ordre & $18 \%$ & $6 \%$ \\
Aide au déroulement & $26 \%$ & $33 \%$ \\
\hline Actes de parole des élèves & $29 \%$ & $27 \%$ \\
\hline
\end{tabular}

Tableau 1 Évolution des actes de parole en classe $(\mathrm{N}=4986)$

Quelques changements peuvent être repérés. Le premier concerne les actes de parole à visée d'instruction qui progressent de 5 points de pourcentage au détriment de ceux dont la visée est éducative. Cette évolution est statistiquement significative (test du $\mathrm{chi}^{2}, p<0,01$. Dans cet article, pour chacun des tests effectués, le seuil de significativité statistique est fixé à 1\%). Ainsi, avec l'expérience, Benoît agit davantage par la parole pour la construction des connaissances mathématiques de ses élèves plutôt que pour leur indiquer des règles de vie de la classe. En ce qui concerne la transmission des savoirs, on constate une augmentation sensible des indications sur les tâches à réaliser (dévolution) et des synthèses 
des savoirs à retenir (institutionnalisation), augmentation qui est assortie à une baisse des aides à la réalisation des tâches (action-formulation) et des évaluations des productions des élèves (validation). Cette évolution est également statistiquement significative (test du $\mathrm{chi}^{2}, p<0,01$ ). La pratique de Benoît s'éloigne donc de celles qui ont été généralement constatés chez les débutants par Butlen, PeltierBarbier et Pézard dans leur recherche déjà citée. Benoît explique davantage ce que doivent faire les élèves en amont, il a moins besoin d'indiquer leurs erreurs et de réexpliquer ce qui est attendu, il prend également plus de temps pour dresser le bilan de ce qui doit être retenu. Le dernier changement repérable est celui de la conduite de la classe : les rappels à l'ordre chutent de manière importante au profit des indications sur l'organisation du cours. L'évolution est statistiquement significative (test du $\mathrm{chi}^{2}, p<0,01$ ), Benoît est bien parvenu à pacifier ses relations avec ses élèves.

Finalement, bien que cette analyse des actes de parole ne puisse rendre précisément compte de la construction des connaissances par les élèves, elle confirme les conclusions précédentes sur la pratique de Benoît : une amélioration de la gestion didactique de l'enseignement qui reste néanmoins largement improvisée, et une attention particulièrement forte à la qualité des relations avec les élèves en classe. Dans le travail de l'équipe codisciplinaire, l'approche psychanalytique montre comment cette posture professionnelle correspond aussi à des nécessités profondes et personnelles de l'enseignant. L'approche sociologique a questionné le fait que cette orientation du métier est loin d'être isolée, elle apporte des analyses du contexte socio-institutionnel qui permettent de comprendre la progression de ce phénomène.

Revenant à l'analyse didactique, nous tenons à souligner combien l'étude des actes de parole montre, par la cohérence qu'elle apporte sur la compréhension de la pratique de Benoît, que les pratiques langagières de l'enseignant constituent bien une composante de sa pratique professionnelle et que leur analyse, dans le cadre d'une recherche, contribue pleinement à celle de cette pratique.

\section{LES ACTES DE PAROLE AU SERVICE DE LA RÉGULATION DU DÉROULEMENT DES SITUATIONS DIDACTIQUES}

Dans la deuxième recherche, l'analyse des actes de parole est partielle, elle concerne essentiellement les échanges entre élèves et enseignant, à l'initiative des élèves. Cette étude permet de montrer comment l'enseignant, dans ces interactions, cherche à influencer et influence effectivement les activités des élèves, et donc comment il intervient, potentiellement au moins, sur leurs apprentissages. Par comparaison à l'étude précédente, celle qui est abordée ici conduit à illustrer le caractère dynamique des interventions des élèves et de l'enseignant, les unes étant déterminées par les autres, et inversement.

Les pratiques enseignantes et les activités des élèves

Dans cette recherche (Roditi 2005), les pratiques de quatre professeurs enseignant la multiplication des nombres décimaux dans des conditions analogues ont été étudiées, toutes les séances portant sur ce thème ont été analysées. Ces enseignants ont été observés dans leur classe de $6^{\mathrm{e}}$ (11 ans), ils avaient chacun au moins une dizaine d'années d'expérience, les classes avaient approximativement le même effectif et étaient composées essentiellement d'élèves de catégorie sociale intermédiaire. Leur niveau mathématique moyen était équivalent d'une classe à l'autre d'après l'évaluation nationale passée en début d'année. Les profes- 
seurs utilisaient le même manuel scolaire et ils ont consacré quatre heures environ à l'enseignement de la multiplication des nombres décimaux, évaluation terminale non comprise.

Nous allons développer ici l'analyse de leur manière d'agir, par le langage, lorsque les élèves ne parviennent pas à réaliser les tâches proposées. Concernant l'activité de l'élève, nous distinguons l'activité potentielle, celle qu'il est censé avoir pour accomplir la tâche demandée ; l'activité réelle, ce qu'il a fait ; et l'activité effective, reconstitution par le chercheur de ce qu'a pu être l'activité réelle, en fonction de l'activité potentielle et des productions recueillies. Le chercheur accède donc à l'activité potentielle par une analyse a priori, et à l'activité effective par une analyse a posteriori; l'activité réelle restant en grande partie inaccessible. Précisons que l'activité potentielle est bien celle qui apparaît comme étant visée par les auteurs du manuel dont la tâche est extraite, et non toutes les activités envisageables par l'analyse a priori. C'est donc le passage de l'activité potentielle déterminée par l'analyse de la tâche à l'activité effective déterminée par l'observation de la classe qui a été particulièrement étudié dans cette recherche interrogeant l'effet des pratiques enseignantes sur les activités mathématiques des élèves.

Pour les besoins de l'étude, les activités potentielles ont été distinguées selon quatre types : le calcul posé, convoquant la technique opératoire, ou instrumenté, c'est-à-dire effectué à la calculatrice ; le calcul mental, raisonné ou approché qui repose sur des techniques variées, des connaissances des nombres et des approximations ; le questionnement théorique qui est une activité de réflexion sur les propriétés de la multiplication (savoir si la multiplication des nombres décimaux reste commutative, si le produit de deux nombres décimaux non nuls est supérieur aux deux facteurs, etc.) ; et, enfin, la résolution de problèmes numériques où les nombres interviennent dans des contextes de la vie sociale, professionnelle ou scientifique.

Les résultats obtenus sont indiqués dans le tableau $n^{\circ} 2$ où les quatre enseignants sont indiqués par la lettre E suivie d'un numéro allant de 1 à 4 .

\begin{tabular}{lllll}
\hline & $E 1$ & E2 & E3 & E4 \\
\hline Activités potentielles & $\mathrm{N}=12$ & $\mathrm{~N}=7$ & $\mathrm{~N}=12$ & $\mathrm{~N}=11$ \\
Calcul posé ou instrumenté & $17 \%$ & $14 \%$ & $17 \%$ & $09 \%$ \\
Calcul mental, ou approché & $58 \%$ & $57 \%$ & $33 \%$ & $55 \%$ \\
Questionnement théorique & $25 \%$ & $29 \%$ & $33 \%$ & $18 \%$ \\
Problème & $00 \%$ & $00 \%$ & $17 \%$ & $18 \%$ \\
\hline Activités effectives & $\mathrm{N}=242$ & $\mathrm{~N}=103$ & $\mathrm{~N}=152$ & $\mathrm{~N}=206$ \\
Calcul posé ou instrumenté & $9 \%$ & $62 \%$ & $27 \%$ & $40 \%$ \\
Calcul mental, ou approché & $58 \%$ & $25 \%$ & $50 \%$ & $44 \%$ \\
Questionnement théorique & $33 \%$ & $13 \%$ & $16 \%$ & $13 \%$ \\
Problème & $00 \%$ & $00 \%$ & $07 \%$ & $03 \%$ \\
\hline
\end{tabular}

Tableau 2 Des activités potentielles aux activités effectives

On notera la différence très importante entre les effectifs des activités potentielles, déterminée a priori à la lecture des exercices proposés, et des activités effectives, déterminées a posteriori à partir des observations effectuées en classe. Cet écart peut paraître surprenant au premier abord, puisque les secondes sont dix à vingt fois plus nombreuses que les premières, nous y reviendrons.

La partie supérieure du tableau ne montre que de faibles différences entre les projets des professeurs, sauf quant aux problèmes faisant référence à un con- 
texte. En revanche, la partie inférieure révèle des disparités significatives quant aux activités effectives (test du chi ${ }^{2}$ de Pearson appliqué sans tenir compte de la ligne des activités de résolution de problèmes dont les effectifs sont pratiquement nuls, $\mathrm{p}<0,01)$.

Les écarts entre les activités effectives n'apparaissent pas au niveau des activités potentielles. Cela s'explique par des pratiques différentes soumises à des contraintes institutionnelles communes. Les enseignants proposent à leurs élèves des exercices globalement analogues, en lien avec les attendus du programme scolaire. En revanche, les mathématiques que ces enseignants induisent en classe diffèrent largement, et cela impacte les activités effectives. Ainsi, comme développé ci-dessous, le même énoncé suivant (correspondant donc à la même activité potentielle) a conduit à des activités effectives très différentes dans les classes observées : «Place la virgule manquante : $1,35 \times 42=5,67$ ». Une brève analyse montre que l'exercice vise à mettre en défaut le comptage des décimales pour provoquer une activité de calcul approché convoquant des ordres de grandeur. Il a été résolu, sur les incitations des différents professeurs, soit comme attendu, soit à la calculatrice, soit encore par des calculs posés.

Les actes de parole des élèves et de l'enseignant,

et leurs effets sur les activités effectives

Puisque les contextes d'enseignement étaient identiques pour les quatre enseignants observés, les décalages sensibles entre activités potentielles et activités effectives s'expliquent par le fait que le contexte socio-institutionnel ne contraint pas totalement leur pratique et qu'en conséquence, ils investissent des marges de manœuvre en cohérence avec leur conception des mathématiques, de leur enseignement et de leur apprentissage. Mais comment, concrètement, interviennent-ils sur les activités des élèves ? Lorsque les enseignants sont sollicités par ces derniers, ils sont amenés à les aider, en les encourageant ou en leur fournissant des indications. C'est d'ailleurs ce que recherchent les élèves qui, eux aussi, agissent sur le monde par le langage...

Les actes de parole des élèves qui portent sur les tâches mathématiques proposées et qui interpellent l'enseignant ont été qualifiés d'incidents didactiques dans la mesure où, justement, ils obligent l'enseignant à réagir (même s'il feint d'ignorer l'incident). Ils se répartissent entre quatre types dominants : les questions, les erreurs, les réponses incomplètes et les silences (lorsqu'un élève interrogé se tait alors que le professeur attend une réponse). Voici, pour les illustrer, des exemples rencontrés à propos de la tâche suivante, proposée par tous les enseignants : «Place la virgule manquante : $1,35 \times 42=5,67$ ».

Question. «Madame, a-t-on le droit de dire qu'il ne manque pas de virgule ?»

Erreur. « Pour placer la virgule, j'ai ajouté un zéro. J'ai écrit : 1,35 ×0,42= 5,67. »

Réponse incomplète. L'élève dit seulement « Pour placer la virgule, j’ai ajouté un zéro. »

Un enseignant pourrait réagir à ces interpellations en invitant à poser l'opération $1,35 \times 42$, il guidera alors les élèves vers une activité technique à l'issue de laquelle ils obtiendront le produit 56,70 . Il pourrait alors inviter ses élèves à mener un calcul raisonné pour en déduire que $1,35 \times 4,2=5,67$. Un autre pourrait demander à ses élèves d'utiliser les ordres de grandeurs, il provoquera alors une activité toute autre. Un troisième pourrait suggérer d'utiliser la calculatrice alors qu'un quatrième pourrait simplement répondre à la sollicitation de l'élève en lui proposant d'y réfléchir davantage et éventuellement d'échanger à ce sujet avec son voisin. Ces quelques exemples aident à comprendre que les activités effectives 
sont à la fois beaucoup plus nombreuses et très différentes des activités potentielles. Ces dernières sont repérées par l'analyse des tâches, elles correspondent, rappelons-le, non pas à toutes celles que permet d'envisager l'analyse a priori, mais bien à celles qui semblent visées par les auteurs du manuel.

En ce qui concerne enfin les actes de parole des enseignants produits en réponse à ces incidents didactiques, plus de $80 \%$ d'entre eux se répartissent selon trois fonctions : corriger l'exercice ou la question, c'est-à-dire apporter la réponse attendue ; guider les élèves vers la démarche souhaitée ; ou relancer l'activité pour un approfondissement de leur réflexion. La section suivante développe les résultats obtenus.

Incidents didactiques et réactions des enseignants

Dans la mesure où les analyses des tâches proposées par les quatre enseignants n'ont pas révélé de différence significative et où les contextes d'enseignement étaient analogues, on pourrait supposer que les actes de parole des élèves concernant les exercices à résoudre n'aient pas été très différents d'une classe à l'autre. Ce serait négliger le caractère interactif des pratiques langagières : si le langage agit sur le monde, le monde, inversement, agit sur le langage. Dans le cas présent, le monde est la pratique enseignante, et nous allons montrer son influence sur les actes de parole des élèves.

\begin{tabular}{lllll}
\hline & $E 1$ & $E 2$ & $E 3$ & $E 4$ \\
\hline Effectifs & $\mathrm{N}=95$ & $\mathrm{~N}=25$ & $\mathrm{~N}=80$ & $\mathrm{~N}=56$ \\
Actes de parole/min. & 3,9 & 2,4 & 5,2 & 2,7 \\
\hline Erreur & $26 \%$ & $28 \%$ & $21 \%$ & $26 \%$ \\
Question & $18 \%$ & $32 \%$ & $15 \%$ & $20 \%$ \\
Réponse incomplète & $36 \%$ & $16 \%$ & $49 \%$ & $36 \%$ \\
Silence & $12 \%$ & $8 \%$ & $6 \%$ & $7 \%$ \\
Autre & $8 \%$ & $16 \%$ & $9 \%$ & $11 \%$ \\
\hline
\end{tabular}

Tableau 3 Les actes de parole des élèves dans les quatre classes observées

Les effectifs des actes de parole des élèves liés aux tâches mathématiques proposées varient dans un rapport de 1 à 4, mais comme les durées d'enseignement ne sont pas identiques (de 3 à 5 heures de cours), nous retenons plutôt la comparaison du nombre d'actes de parole par minute. Des différences sensibles apparaissent également : dans la classe de l'enseignant E3, les élèves interviennent deux fois plus que dans celle de l'enseignant E2.

L'examen de la répartition des actes de parole est également instructif. Ils apparaissent avec environ la même fréquence dans les quatre classes sauf pour trois valeurs qui s'écartent de la tendance générale : les questions sont très fréquentes dans la classe de $\mathrm{E} 2$, au détriment des réponses incomplètes, ces dernières étant, au contraire, prépondérantes chez E3. Les pratiques de ces deux enseignants éclairent ces valeurs atypiques. Le croisement des observations et des faits statistiques montrent que E3 encourage et valorise l'expression de ses élèves, ce qui les conduit à répondre, même lorsque leur réflexion n'est pas aboutie. E2, en revanche, critique facilement les élèves qui se trompent, aussi, quand ils ne sont pas sûrs d'eux, au lieu de répondre de façon incomplète, ils préfèrent questionner leur professeur afin d'obtenir des indications complémentaires qui leur permettront de trouver la réponse attendue. C'est, rappelons-le, dans la classe de E3 et E2 que les élèves interviennent respectivement le plus et le moins. 
L'analyse des actes de parole des enseignants conduit d'abord à comparer leurs manières de répondre aux interventions des élèves.

\begin{tabular}{lllll}
\hline & $E 1$ & $E 2$ & $E 3$ & $E 4$ \\
\hline Effectifs & $\mathrm{N}=89$ & $\mathrm{~N}=25$ & $\mathrm{~N}=77$ & $\mathrm{~N}=46$ \\
\hline Corriger & $24 \%$ & $56 \%$ & $57 \%$ & $37 \%$ \\
Guider & $13 \%$ & $0 \%$ & $17 \%$ & $13 \%$ \\
Relancer & $46 \%$ & $12 \%$ & $18 \%$ & $35 \%$ \\
Autres & $17 \%$ & $32 \%$ & $8 \%$ & $15 \%$ \\
\hline
\end{tabular}

Tableau 4 Les actes de parole des quatre enseignants observés

On peut remarquer d'abord que les actes de parole des enseignants sont légèrement moins nombreux que ceux des élèves, cela s'explique par le fait que, parfois, des élèves interviennent simultanément. L'examen du tableau $n^{\circ} 4$ révèle que les actes de parole varient de manière significative d'un enseignant à l'autre (test de $\mathrm{chi}^{2}, \mathrm{p}<0,01$ ). Ainsi E2 et E3 corrigent davantage, c'est-à-dire prennent davantage à leur charge la réalisation de la tâche proposée, alors que E1 et E4 privilégient la relance de l'activité des élèves.

Une analyse complémentaire, plus délicate, a été menée pour savoir si, chez un même enseignant, la manière de réagir aux interventions des élèves était ou non indépendante de la nature de ces interventions. Pour répondre à cette question, nous avons comparé, d'une part, les effectifs des types d'actes de parole de l'enseignant associés aux types d'intervention des élèves, et, d'autre part, les effectifs qui auraient été observés si les actes de parole de l'enseignant n'étaient pas influencés par les interventions des élèves. Voici par exemple le tableau de contingence des actes de paroles des élèves et de l'enseignant E1. Dans chaque case du tableau, les valeurs observées sont indiquées à gauche, alors qu'entre parenthèses et à droite, ont été portés les effectifs qu'on aurait obtenus si les actes de paroles de l'enseignant E1 et des élèves avaient été indépendants. Ces effectifs se calculent à partir de la dernière ligne et de la dernière colonne du tableau. Les valeurs observées sont en italiques lorsqu'elles présentent un écart jugé important avec les valeurs théoriques (plus de $20 \%$ de l'effectif théorique, pour ceux qui sont supérieurs à 3 ).

\begin{tabular}{llllllc}
\hline & Erreur & Question & Incompl. & Silence & Autre & Total \\
\hline Corriger & $5(5,4)$ & $6(3,8)$ & $6(7,6)$ & $1(2,6)$ & $3(1,7)$ & 21 \\
Guider & $1(3,1)$ & $1(2,1)$ & $6(4,3)$ & $4(1,5)$ & $0(0,9)$ & 12 \\
Relancer & $14(10,6)$ & $5(7,4)$ & $18(14,7)$ & $2(5,1)$ & $2(3,2)$ & 41 \\
Autres & $3(3,9)$ & $4(2,7)$ & $2(5,4)$ & $4(1,8)$ & $2(1,2)$ & 15 \\
Total & 23 & 16 & 32 & 11 & 7 & 89 \\
\hline
\end{tabular}

Tableau 5 Contingence des actes de parole de l'enseignant E1 et de ses élèves

Ce travail permet une analyse fine des modes de gestion des incidents. Ainsi, la première colonne montre que lorsque l'enseignant E1 est confronté à un acte de parole correspondant à une erreur, plus qu'il ne le fait en général, il agit par le langage pour relancer le travail le travail de ses élèves. Les autres valeurs du tableau indiquent également que l'enseignant E1 corrige davantage qu'il ne le fait d'ordinaire lorsque les élèves posent des questions, mais évite de le faire lorsqu'ils donnent des réponses incomplètes, il préfère dans ce cas relancer ou les guider. Il privilégie ainsi la relance lorsque les élèves commettent des erreurs ou qu'ils produisent des réponses incomplètes, et délaisse ce moyen vis-à-vis de ceux qui le 
questionnent ou qui restent silencieux lorsqu'ils sont interrogés. La description de la pratique (langagière) de E1 s'affine : il a plutôt tendance à relancer l'activité des élèves, sans imposer trop de tension à ceux qui l'interrogent ou qui ne savent pas quoi dire, mais en étant particulièrement exigeant avec ceux qui se trompent ou qui ne répondent pas complètement.

Les analyses analogues menées pour les autres enseignants conduisent à des conclusions différentes. Finalement, malgré les contraintes socio-institutionnelles qui pèsent sur l'enseignement, il reste des marges de manœuvre que les enseignants investissent personnellement; ils influencent alors les activités mathématiques effectives de leurs élèves, y compris leurs interventions en classe. La dernière recherche étudie l'effet des pratiques enseignantes, non plus sur les activités des élèves, mais directement sur leur apprentissage.

\section{EFFETS DES ACTES DE PAROLE DE L'ENSEIGNANT SUR L'APPRENTISSAGE DES ÉLÈVES}

Cette dernière recherche porte sur les aides qu'un enseignant peut apporter à ses élèves en difficulté pour comparer des nombres décimaux. Nous allons ici essentiellement examiner le rôle que certains de ses actes de parole peuvent jouer sur l'apprentissage. Le lecteur intéressé par la recherche complète peut en consulter sa publication (Roditi 2007).

Les élèves qui butent sur la comparaison de deux décimaux mettent en œuvre des règles implicites et erronées qui ont été élucidées depuis plus de trente ans (Grisvard \& Leonard 1981, 1983). La plus fréquemment utilisée conduit à écrire que 8,14 est supérieur à 8,7 au motif que 14 est supérieur à 7. D'autres travaux ont été menés depuis en didactique des mathématiques, si bien que nous disposons d'un corpus de connaissances solides et précises sur les questions d'enseignement et d'apprentissage des nombres décimaux (Perrin-Glorian 1984 ; Douady \& Perrin-Glorian 1986 ; Brousseau 1998). Dans notre recherche, grâce à ces connaissances, un questionnaire a été conçu qui a été administré à plus de 400 élèves, une centaine pour chacun des quatre niveaux suivants : CM2 (10 ans), $6^{\mathrm{e}}$ (11 ans), $5^{\mathrm{e}}$ (12 ans) et BEP (15 ans). Il a permis d'en repérer 58 particulièrement en difficulté parmi ceux des trois niveaux inférieurs (leur score de réussite étant de plus d'un écart type inférieur au score moyen) et de leur proposer de participer à un dispositif d'aide portant notamment sur les représentations des nombres et sur l'explicitation des méthodes de comparaisons.

\section{La comparaison des décimaux est plus difficile à l'oral qu'à l'écrit}

Le questionnaire comportait deux parties qui ont été passées par les élèves lors de deux jours différents. Cela nous a permis de leur proposer des comparaisons de deux décimaux portant sur les mêmes nombres, mais présentés une fois par écrit et l'autre fois oralement. Par exemple, on demandait à l'écrit d'entourer le plus grand des nombres entre 8,7 et 8,14 alors qu'oralement on demandait d'écrire le plus grand des deux nombres. Des comparaisons en contexte ont également été proposées, par écrit ou oralement. Il s'agissait par exemple d'énoncés du type : «Manu mesure 1,21 m. Léa mesure 1,4 m. Qui est le plus grand ? » Les résultats montrent que la présentation orale accroît la difficulté. Le pourcentage de réponses erronées subit en effet un accroissement significatif (de $5 \%$ à $11 \%$ ) lorsqu'on passe des questions où les nombres sont proposés par écrit à celles où ils sont donnés oralement (test $\left.\mathrm{du}^{\mathrm{chi}}{ }^{2}, \mathrm{p}<0,01\right)$. Le constat est ana- 
logue chez les 58 élèves du groupe faible : le pourcentage d'erreurs passe alors de $29,2 \%$ à $41,4 \%$.

Ces chiffres rendent compte des difficultés que cause la langue française orale qui associe, à la partie décimale, la même expression langagière qu'à la partie entière (huit virgule quatorze pour 8,14 ) alors que d'autres langues, comme l'anglais, distinguent la manière d'oraliser ces deux parties (huit point un quatre pour 8.14) sauf quand la partie décimale est associée à une sous-unité qui doit alors être explicitée (huit livres et quatorze cents pour $8.14 £$ ).

À la lumière de ces résultats et de ceux qui ont été produits par les autres recherches déjà mentionnées, trois axes principaux ont été retenus pour concevoir notre dispositif d'aide : proposer des situations écrites où les nombres décimaux peuvent être lus et pas seulement entendus ; favoriser un traitement sémantique des écritures numériques s'appuyant sur différents registres de représentation; faire expliciter et critiquer des procédures de comparaison. C'est sur ce dernier aspect que nous voudrions insister dans ce texte.

\section{Des contre-suggestions}

pour provoquer l'explicitation des procédures des élèves

Le dispositif qui a été mis en place par un enseignant concerne les élèves ayant pu y participer parmi les 58 qui avaient été jugés les plus faibles. Ces élèves sont pris en entretiens individuels d'une quarantaine de minutes. Le dispositif commence par un court questionnaire de comparaisons de deux décimaux afin de travailler avec l'élève à partir de ses propres productions. Après chaque réponse, il est invité à revenir sur la tâche et à représenter concrètement les nombres comparés à l'aide de différents moyens : des pièces de monnaie, des graduations, des surfaces quadrillées, etc. Il doit également expliciter et critiquer ou argumenter des procédures de comparaison. Ce travail supplémentaire associé à la même tâche est demandé par l'enseignant qui prend en charge l'aide.

Autrement dit, le dispositif mis en place propose à des élèves qui, l'enseignant le sait, échouent massivement dans la réalisation d'une tâche de comparaison, de réaliser quand même cette tâche, mais accompagnés par l'enseignant, et en s'appuyant sur du matériel ayant une fonction symbolique importante relativement aux savoirs en jeu. Ce dispositif rappelle celui de la double-stimulation mis en œuvre par Vygotsky et Leontiev. Comme l'explique Rochex $(1997,120)$, «celleci consiste à proposer à l'enfant des tâches (stimuli-objets) qui se situent un peu au-delà de ses capacités présentes, qu'il ne peut donc résoudre seul, puis à lui fournir des auxiliaires extérieurs (stimuli-instruments ou stimuli-signes) de nature à l'aider dans la réalisation de la tâche parce que lui permettant un meilleur contrôle de son comportement ».

Parce que le langage représente un des leviers majeurs du pari engagé dans cette recherche pour mettre les connaissances didactiques au service des élèves à travers ce dispositif d'aide, nous souhaitons insister sur le choix et la portée des actes de parole produits par l'enseignant. Ils ont été programmés en amont de la mise en œuvre du dispositif pour être adaptés à chaque réponse de l'élève et lui permettre ainsi de travailler au mieux sur les trois registres simultanément: l'expression écrite et orale des nombres décimaux ; leurs représentations concrètes (et non plus symbolique); les discours qui expriment les règles de comparaison et leur justification.

À l'élève qui doit comparer 8,7 et 8,14 et qui propose le nombre correct, l'enseignant dit précisément : "L'autre jour, un élève comme toi avec qui je travaillais, m'a dit que comme 7 est plus petit que 14, on peut en déduire que 8,7 est 
plus petit que 8,14. Qu'est-ce que tu en penses ? Comment pourrais-tu faire pour voir qui a raison avec le matériel qui est là ? ». Il lui propose donc, alors qu'il a répondu correctement, de confronter sa réponse à celle d'un élève fictif qui tiendrait un raisonnement faux, mais très courant, aboutissant à un résultat différent du sien. Si, en revanche, l'élève ne répond pas correctement et propose le mauvais nombre, l'enseignant tente de lui faire expliciter la démarche qu'il a utilisée. Dans le cas où l'élève ne parvient pas à expliciter sa procédure, l'enseignant lui suggère la règle implicite qu'il a certainement utilisée, c'est-à-dire exactement celle qui a été proposée précédemment : "Un élève m'a expliqué que comme 7 est plus petit que 14, on peut en déduire que 8,7 est plus petit que 8,14. Qu'est-ce que tu en penses? ». L'élève ayant reconnu sa démarche, l'enseignant lui propose la contresuggestion suivante, celle d'un élève fictif qui tiendrait un raisonnement correct et qui aboutirait donc à une autre réponse que celle annoncée: «Un autre élève, comme toi avec qui je travaillais m'a dit que 7 dixièmes c'est pareil que 70 centièmes, et que comme 70 est plus grand que 14, c'est 8,7 qui est plus grand que 8,14. Qu'est-ce que tu en penses? Comment pourrais-tu faire, avec le matériel qui est là, pour voir qui a raison? ». Puis l'enseignant demande à l'élève de mettre des mots sur ses manipulations matérielles, sur les conséquences qu'il en tire ainsi que sur les généralisations qu'il peut en déduire.

Cette idée d'interpeler les élèves à l'aide de contre-suggestions nous vient de la lecture de Piaget, néanmoins, on le comprend, notre démarche est inspirée par les travaux de Vygotski (1934/1997) sur l'acquisition des savoirs scientifiques. Par un travail précis avec et sur du langage mathématique employé dans l'intersubjectivité entre l'enseignant et l'élève, nous pensons agir pour que ce dernier fasse siennes les règles correctes de comparaisons des nombres décimaux, en ayant compris leur fondement, et en ayant compris aussi pourquoi celles qu'il utilisait implicitement auparavant sont inexactes.

Une évaluation des progrès des élèves ayant pu participer à ce dispositif d'aide a été réalisée. Onze élèves seulement parmi les 58 du groupe faible ont pu y participer car nous avons rencontré des difficultés à faire autoriser un travail en établissement scolaire en dehors des heures de cours... Alors que le questionnaire final comportait les mêmes difficultés que le questionnaire initial quant à la comparaison de deux nombres décimaux, le taux d'erreurs sur ces questions est passé de 35,6\% au premier questionnaire à $0,6 \%$ au second ! Ainsi, des tâches précisément conçues et des actes de parole finement adaptés aux difficultés des élèves semblent pouvoir contribuer à les aider à progresser de façon significative (test du $\left.\mathrm{chi}^{2}, \mathrm{p}<0,01\right)$. Une question reste cependant posée, celle de la contribution de la conception des tâches et des actes de parole adaptés à l'efficacité du dispositif. On ne peut négliger, en effet, que tout temps de travail supplémentaire proposé à un élève est susceptible d'améliorer ses apprentissages. La durée relativement courte des aides apportées ainsi que le caractère généralement persistant des difficultés rencontrées sur la comparaison des nombres décimaux, malgré l'interrogation qui demeure sur le plan scientifique, nous invitent néanmoins à supposer que le dispositif mis en œuvre est davantage favorable aux progrès des élèves qu'une «aide personnalisée » ou une « aide aux devoirs » telles qu'elles sont généralement proposées en milieu scolaire, c'est-à-dire sans lien avec une analyse didactique fine des enjeux d'apprentissage et des origines des difficultés. 


\section{CONCLUSION}

La même option théorique fonde ces trois recherches : le langage est un moyen, pour l'enseignant, d'agir sur l'activité des élèves et, par conséquent, sur leurs apprentissages. En ce sens, l'analyse des pratiques langagières des enseignants contribue à celle, plus globale, de leur pratique.

Dans chaque recherche, les méthodes utilisées reposent sur une classification des actes de parole des enseignants spécifiquement adaptée à la problématique et correspondant toujours à des fondements théoriques didactiques. Dans l'étude de l'évolution de la pratique de Benoît, nous avons retenu d'une part l'objectif poursuivi, entre éducation et transmission des savoirs, et d'autre part la fonction quant à la dévolution, à l'action et à la formulation des élèves, à la validation de leurs productions ou à l'institutionnalisation des savoirs. Dans l'étude comparative des pratiques d'enseignement de la multiplication des décimaux, nous avons plutôt focalisé notre attention sur les réactions langagières des enseignants à certains actes de parole de leurs élèves (les incidents didactiques) et nous avons distingué ces réactions en fonction de leur effet visé sur l'activité des élèves. La dernière recherche sur l'aide à la comparaison des nombres décimaux a davantage approfondi et évalué une manière particulière (adaptée au contenu mathématique visé) de proposer des outils symboliques pour aider l'élève à réaliser une tâche qu'il ne peut réaliser seul et d'adapter les actes de parole aux difficultés réellement rencontrées par cet élève à qui ils sont adressés pour en évaluer l'effet sur l'apprentissage.

La didactique des mathématiques est fondée, depuis son origine, sur une conception constructiviste et interactionniste du sujet élève. Cela constitue l'une des raisons qui conduisent les didacticiens vers l'étude des questions langagières, d'autres tiennent notamment au fait que les mathématiques elles-mêmes reposent sur un langage spécifique. Aussi les recherches en didactique des mathématiques convoquent-elles, plus ou moins directement, des outils construits par des linguistes. Les échanges entre didacticiens et linguistes contribuent à un développement et à un renforcement de leur champ de recherche respectif, parce qu'ils constituent des objets scientifiques différents sur des réalités communes : les pratiques langagières des élèves et des enseignants.

Éric RODITI

Université Paris Descartes

Laboratoire EDA (Éducation Discours Apprentissages)

eric.roditi@paris5.sorbonne.fr

\footnotetext{
Abstract : This article uses three pieces of research in the field of mathematics education in order to offer several ways to analyse teachers'practices by analysing teachers' discourses. Its theoretical framework leads to consider teachers'discourses as an ingredient rather than a product of teaching practices. Although linguistic concepts and tools can help to offer a certain perspective, the central issue is to understand how teaching practices contribute to pupils' learning.

Keywords : teachers'practices, decimal numbers, geometry, speech acts, didactic and ergonomic approach.
} 


\section{Références}

Austin J. L. (1970) Quand dire c'est faire. Paris : Le Seuil.

Bulten D, Peltier-Barbier M.-L. et Pézard M. (2002) « Nommés en REP, comment font-ils ? Pratiques de professeurs d'école enseignant les mathématiques en REP : contradiction et cohérence »-RFP 140 (41-52).

Brousseau G. (1998) Théorie des situations didactiques. Grenoble: La Pensée Sauvage.

Chareaudeau P. \& Maingueneau D. (dir.) (2002) Dictionnaire d'analyse du discours. Paris : Le Seuil.

Chaussecourte P. (dir.) (2014) Enseigner à l'école primaire. Dix années avec un professeur des écoles. Paris : L'Harmattan.

Douady R. \& Perrin M.-J. (1986) Liaison école-collège: Nombres décimaux. Brochures de l'IREM de Paris VII 62.

Grisvard C. \& Leonard F. (1981) «Sur deux règles implicites utilisées dans la comparaison de nombres décimaux positifs »-Bulletin de l'APMEP 327 (87-60).

Grisvard C. \& Leonard F. (1983) «Résurgence de règles implicites dans la comparaison de nombres décimaux »-Bulletin de l'APMEP 340 (450459).

Kerbrat-Orecchioni C. (1990) Les Interactions verbales. Paris : Armand Colin.

Leontiev A. (1984) Activité Conscience Personnalité. Moscou : Éditions du Progrès.

Leplat J. (1997). Regard sur l'activité en situation de travail. Contribution à la psychologie ergonomique. Paris : PUF.

Nuchèze de V. (2001) Sémiologie des dialogues didactiques. Paris : L'Harmattan.

Peltier-Barbier M.-L. (dir). (2004) Dur d'enseigner en ZEP. Grenoble : La Pensée Sauvage.

Perrin M.-J. (1984) «Représentation des fractions et des nombres décimaux chez des élèves de CM2 et du collège »-Cahiers de Didactique des Mathématiques de l'IREM de Paris VII, 24.

http://www.irem.univ-paris-diderot.fr/up/CDM_24_Marie_jeanne_Perrin__Représentation_des_fractions_et_des_nombres_décimaux_ches_ds_élèves_de_CM2 _et_du_collège.pdf

Robert A. \& Rogalski J. (2008) «Cadrages théoriques »- in : F. Vandebrouck (coord.) La classe de mathématiques (9-30). Toulouse : Octares.

Rochex J.-Y. (1997) «L'oeuvre de Vygotski : fondements pour une psychologie historico-culturelle »-RFP 120 (105-147)

Roditi E. (2005) Les pratiques enseignantes en mathématiques. Entre contraintes et liberté pédagogique. Paris : L'Harmattan.

Roditi E. (2007) «La comparaison des nombres décimaux, conception et expérimentation d'une aide aux élèves en difficulté »-Annales de Didactique et de Sciences Cognitives 12 (55-81).

Roditi E. (2013) «Une orientation théorique pour l'analyse des pratiques enseignantes en didactique des mathématiques »-Recherches en Didactiques 15 (39-60).

Searle J. (1972) Les actes de langage. Paris : Hermann.

Vandebrouck F. (dir.). (2008) La classe de mathématiques : activités des élèves et pratiques des enseignants. Toulouse : Octarès.

Vygotski L. (1934/1997) Pensée et langage. Paris : La Dispute. 